\title{
RTT Evaluation of a novel precision template-guided biopsy system for detecting prostate cancer
}

\author{
Ifeanyichukwu I. Megwalu*, Genoa G. Ferguson*, John T. Wei', \\ Vladimir Mouraviev ${ }^{\ddagger}$, Thomas J. Polascik ${ }^{\ddagger}$, Samir Taneja ${ }^{\S}$, Linda Black*, \\ Gerald L. Andriole ${ }^{* \uparrow}$ and Adam S. Kibel ${ }^{* \uparrow}$
}

*Division of Urology, "Siteman Cancer Center, Washington University School of Medicine, St. Louis, MO, ${ }^{+}$Department of Urology, University of Michigan School of Medicine, Ann Arbor, MI, ${ }^{\ddagger}$ Division of Urology, Duke University School of Medicine, Durham, NC, and ${ }^{\S}$ Department of Urology, New York University School of Medicine, New York, NY, USA Accepted for publication 28 April 2008

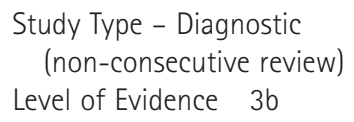

\section{OBJECTIVE}

To explore the ability of a novel transrectal ultrasonography (TRUS) device (TargetScan ${ }^{\mathrm{TM}}$, Envisioneering Medical Technologies, St. Louis M0) that creates a three-dimensional map of the prostate and calculates an optimal biopsy scheme, to accurately sample the prostate and define the true extent of disease, as standard TRUS-guided prostate biopsy relies on the operator to distribute the biopsy sites, often resulting in under- and oversampling regions of the gland.

\section{PATIENTS AND METHODS}

In a multicentre retrospective chart review evaluating patients who had a TargetScan prostate biopsy between January 2006 and June 2007, we determined the overall cancer detection rate in all patients and in subgroups based on prostate specific antigen level, digital rectal examination, and indication for biopsy. We assessed the pathological significance of cancer detected, defined as a Gleason score of $\geq 7$, positive margins, extracapsular disease or $>20 \%$ tumour volume in the prostatectomy specimen. We also evaluated the concordance in Gleason score between the biopsy and prostatectomy specimen.

\section{RESULTS}

Cancer was detected in 50 (35.7\%) of the 140 patients biopsied, including 39 (47.6\%) with no previous biopsies. Of 23 prostatectomy specimens, 20 (87\%) had pathologically significant disease. The biopsy predicted the prostatectomy Gleason score in 12 patients (52\%), overestimated in two (9\%), underestimated in eight (35\%), and biopsy Gleason score could not be assigned in one (4\%).

\section{CONCLUSIONS}

Template-guided biopsy potentially produces a higher cancer detection rate and more accurate assessment of grade. Prostatectomy specimens did not have a high rate of pathologically insignificant disease.

\section{KEYWORDS}

prostatic neoplasms, diagnosis, biopsy, sampling

\section{INTRODUCTION}

Prostate cancer is the most common solid malignancy in men, with an estimated 218890 new cases and 27050 deaths in 2007 [1]. Screening with PSA level and a DRE has led to an increase in detection, and decrease in disease-specific mortality [2]. TRUS-guided prostate biopsy has been the standard method for diagnosing prostate carcinoma, but has several potential shortcomings.

False-negative rates remain of concern, with estimates that office-based TRUS-guided biopsy misses $\approx 30 \%$ of clinically significant prostate cancer [3], and a high percentage
(23\%) of cancers missed are high-grade (Gleason 8 and 9) [4]. In addition, among those in whom prostate cancer is detected, the manner in which the biopsy cores are obtained makes it difficult to precisely characterize the true extent of the tumour. Standard manually guided biopsy sampling might make Gleason scores or needle-core volumes imprecise. As both correlate with outcome after therapy [5], an accurate assessment of tumour characteristics is critical for counselling patients and guiding therapy.

The primary technique to minimize falsenegative rates of TRUS-guided biopsy has been to take more cores per biopsy session, and include more laterally placed biopsy cores to sample the peripheral zone [6-8]. While this has decreased the under-sampling of tumours, it has associated morbidity $[9,10]$, which increases as more cores are taken during biopsies [11]. Also there is increasing concern that patients are being diagnosed with clinically insignificant disease [12].

Given these limitations of contemporary biopsy schemes, there is a motivation to develop an improved instrument or diagnostic method that has a high detection rate for prostate cancer within the 'window of opportunity' for treatment, but minimizes the 
FIG. 1. Biopsy Scheme - A) Sagital view demonstrates that needles are placed uniformly from apex to base. B) Transverse view demonstrates that needles are placed uniformly from right to left.
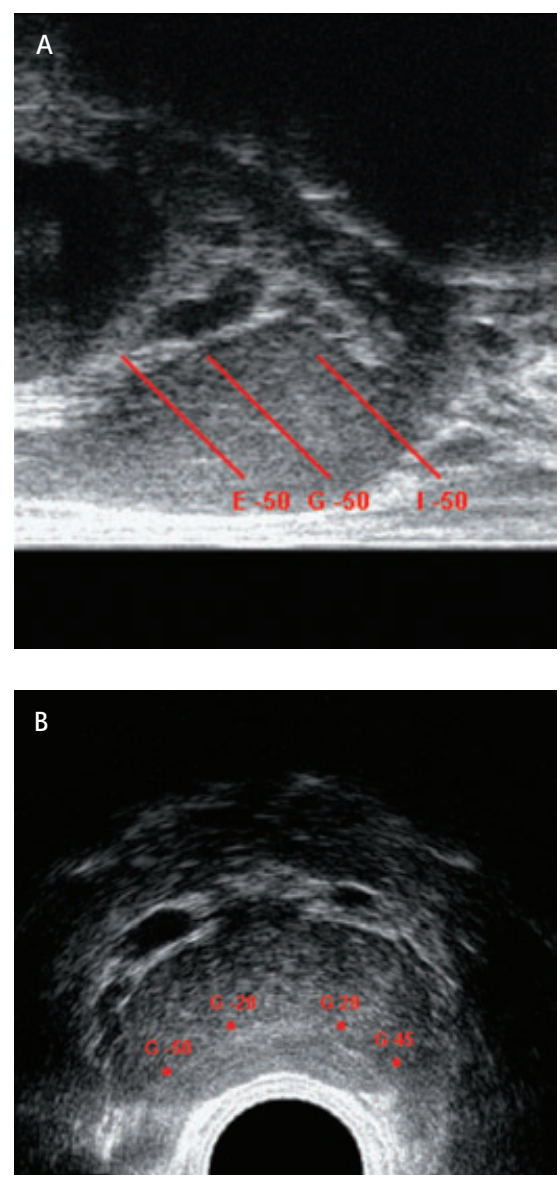

detection of clinically insignificant disease. Recently, there have been many new techniques for prostate cancer diagnosis, including the use of ultrasonographic contrast agents, colour Doppler, power Doppler and MR spectroscopy image-directed TRUS-guided biopsy $[4,13,14]$.

The TargetScan ${ }^{\mathrm{TM}}$ system (Envisioneering Medical Technologies, St. Louis, MO, USA) is a novel TRUS device that allows accurate needle placement within a template. First, a threedimensional map of the prostate is created; then a computer algorithm calculates an optimum biopsy scheme using the measured dimensions of the prostate. The system then uses a fixed template that allows the physician to biopsy the prostate at specific locations. The instrument can be used for 12 core template biopsy or for targeted and saturation biopsy if indicated. The instrument can be used to target the same region of the prostate in the future if needed, which is particularly useful in patients with suspicious histology, such as atypia, on initial biopsy.

The aim of the present study was to describe a multicentre experience with the TargetScan template-guided biopsy system, and to show how improved precision potentially translates into a higher cancer detection rate without leading to increased detection of pathologically insignificant disease.

\section{PATIENTS AND METHODS}

In a multicentre retrospective chart review, after obtaining institutional review board approval at all sites, we retrospectively reviewed the clinical and pathological data of 199 consecutive patients at Washington University, Duke University, and University of Michigan who had a prostate biopsy using the TargetScan system between January 2006 and June 2007. We excluded patients who did not have PSA or DRE findings recorded (two), those who had had a previous diagnosis of prostate cancer (13), and those who were enrolled in a prospective evaluation of the device (44); the final study population comprised 140 patients.

The data extracted from charts included the most recent serum PSA level, DRE findings, number of previous biopsies, pathology from biopsies, and, if performed, surgical pathology from the radical prostatectomy (RP).

Pathological variables recorded were Gleason score, volume of disease, margin status, invasion of seminal vesicles, lymph node status, extracapsular extension and perineural invasion. Pathological information was collected from surgical pathology reports, and clinical information, including PSA and DRE findings, were collected primarily from a form completed by the treating physician on the day of the procedure. Other clinical information, including patient age and number of previous biopsies, were obtained from all other documents available in patients' medical records.

The TargetScan system is a commercially available TRUS template-guided prostate biopsy instrument approved by the USA Food and Drug Administration. The instrument has the following components: An endorectal ultrasound probe, which once inserted in the rectum remains fixed for the entire procedure; an ultrasound transducer which moves within the probe to provide, within 1-2 min, a scan of the prostate created in $1-\mathrm{mm}$ increments, thus increasing image precision; a PC-based digital hardware and software system for image planning, data storage and retrieval; a novel single use flexible $18 \mathrm{G}$ Nitinol biopsy needle which is inserted into the biopsy guide parallel to the probe, but exits at $45^{\circ}$, thus maximally sampling the peripheral zone.

Using the TargetScan is an office-based procedure, performed under local anaesthesia with patients in the dorsal lithotomy position. The probe is inserted transrectally; the ultrasonograms are taken and can be displayed simultaneously in transverse and sagittal planes, and reconstructed in three dimensions. While the instrument has the capacity for targeted biopsy and saturation biopsy, in our experience, a 12-core template biopsy was used. The system plans a 12-core biopsy using the following algorithm: from the sagittal view, three planes that are equally spaced with respect to prostate apex and base are calculated; at each of the three preselected transverse planes, the left and right edges of the prostate are marked and four locations are calculated for each plane (Fig. 1). The position of the biopsy guide is quantified using depth of insertion ( $\mathrm{cm}$ from the apex) and rotational variables (degrees from the 12 o'clock position). The precise location from which samples were taken is stored using the two co-ordinates, and is included in a printed report. The entire procedure lasts $\approx 10 \mathrm{~min}$ for a 12-core template biopsy.

We determined the overall cancer detection rate in all patients requiring biopsy, and then in various subgroups based on serum PSA level, DRE findings, and the indication for biopsy. We assessed the pathological significance of cancer detected by evaluating surgical pathology reports in patients who had RP. We defined pathological significance as a Gleason score of $\geq 7$, extracapsular disease, positive margins, or $>20 \%$ tumour volume in the RP specimen.

\section{RESULTS}

The study comprised 140 men (median age 64 years, range 46-85); 107 received care at Washington University, 22 at Duke University, and 11 at the University of Michigan. All patients had a DRE and serum PSA testing. The median (range) serum PSA level in all patients was $5.2(0.4-87.5) \mathrm{ng} / \mathrm{mL}$. Fifty-eight 
patients (41.4\%) had had previous six- to 12core biopsies using the standard technique.

The template biopsy detected prostate cancer in 50 of the 140 patients (35.7\%) and 39 of 82 $(48 \%)$ having their first biopsy. The cancer detection rate in subgroups based on the indication for biopsy is shown in Table 1 , which also shows the percentage of cancerpositive biopsies based on the number of previous biopsies. Table 2 shows the prostate cancer detection rate in groups stratified by PSA level and DRE findings.

In all, 23 of $50(46 \%)$ patients had a RP; the median (range) Gleason sum score was 7 (6-9) and median volume of disease was $10(1-30) \%$. Of the 23 patients, nine (39\%) had a positive margin, two (9\%) had invasion of the seminal vesicle, none had positive lymph nodes, seven (30\%) had extracapsular extension and 13 (57\%) had perineural invasion. Based on the definition of pathologically significant disease (Gleason score $\geq 7$, extracapsular extension, positive margins, or $>20 \%$ tumour volume), only three patients (13\%) were found to have pathologically insignificant disease.

Comparing the Gleason score from the biopsy cores with that from the RP specimen showed concordance in 12 of the 23 patients (52\%), over-grading in two (9\%), under-grading in eight (35\%), and in one patient (4\%), insufficient tissue was obtained on biopsy to assign a Gleason score, but the patient was later assigned Gleason 6 disease after RP.

\section{DISCUSSION}

Prostate cancer is the most common malignancy in men [1]. Given the need to detect the cancer during the 'window of opportunity' for treatment, but also the concurrent need to minimize the detection of clinically and pathologically insignificant disease, there has been an increasing search for newer techniques to satisfy these demands. In our experience with the TargetScan device, $48 \%$ of patients having their first biopsy and 36\% of the entire group were positive for prostate cancer. This is higher than has been observed with traditional TRUS-guided 12-core biopsy, at 27-42\%. For example, Gore et al. [15] detected cancer in 139 of 375 (37.1\%) of all patients undergoing biopsy, and 112 of 264 (42.4\%) patients having their first biopsy. Presti et al. [8] and Guichard et al. [16], after

TABLE 1 The cancer detection rate in subgroups (two patients did not have reason for biopsy recorded) and based on the number of previous biopsies

\begin{tabular}{lccc} 
Reason for biopsy & $\begin{array}{l}\text { No. of cancers } \\
\text { detected }\end{array}$ & $\begin{array}{l}\text { Total no. of } \\
\text { biopsies }\end{array}$ & $\begin{array}{l}\text { Positive biopsies } \\
\% \text { or } n / N\end{array}$ \\
\hline Elevated PSA level only & 35 & 102 & 34.3 \\
Abnormal DRE only & 3 & 10 & $3 / 10$ \\
Elevated PSA+ abnormal DRE & 9 & 12 & 69 \\
History of atypia on previous biopsy & 1 & 14 & $1 / 14$ \\
Biopsy number & & & \\
First & 39 & 82 & 47.6 \\
Second & 6 & 23 & 27.3 \\
Third & 3 & 14 & $3 / 14$ \\
Fourth & 1 & 8 & $1 / 8$ \\
Fifth & 0 & 8 & 0 \\
Sixth & 1 & 4 & $1 / 4$ \\
Seventh & 0 & 1 & 0
\end{tabular}

TABLE 2 Prostate cancer detection rates stratified by PSA level and DRE findings

\begin{tabular}{|c|c|c|c|c|c|}
\hline \multirow[b]{2}{*}{ DRE } & \multicolumn{4}{|c|}{ Positive for cancer/total, $n / N(\%)$, at PSA, ng/mL } & \multirow[b]{2}{*}{ Total } \\
\hline & $\overline{<2.5}$ & $2.6-4.0$ & $4.1-10.0$ & $>10.0$ & \\
\hline Negative & $1 / 7$ & $3 / 17(18)$ & $22 / 68(32)$ & $6 / 12$ & $32 / 104(30.8)$ \\
\hline Positive & $2 / 9$ & $2 / 3$ & $12 / 22(55)$ & $2 / 2$ & $18 / 36(50)$ \\
\hline Total & $3 / 16(19)$ & $5 / 20(25)$ & $34 / 90$ (38) & $8 / 14$ & $50 / 140(36)$ \\
\hline
\end{tabular}

evaluating patients having their first biopsy, found a cancer detection rate of $44.4 \%$ and $38.7 \%$, respectively; in both studies, patients had a 12-core biopsy. Some studies have reported markedly lower cancer detection rates; e.g. Naughton et al. [17] reported a cancer detection rate of only $27 \%$ in their entire group of patients having a 12-core biopsy. While the present study implies that the TargetScan improves detection rates, it is difficult to compare cancer detection rates between these studies, given that there are almost certainly differences in the populations studied.

Not surprisingly, when we assessed subgroups with different indications for biopsy (Table 1), the highest cancer detection rate was in those having a biopsy for both an elevated PSA level and an abnormal DRE, followed by the group biopsied for an elevated PSA level only, then the group biopsied for abnormal DRE findings only, and finally those biopsied because they had a history of pathological atypia on previous biopsy. As shown in Table 2, when groups were stratified by both PSA and DRE findings, the highest cancer detection rate was in the subgroup with a positive DRE and
PSA level of $>10 \mathrm{ng} / \mathrm{mL}$. As shown in Table 2, as the PSA level increased, the rate of cancer detection increased both in those with a positive and those with a negative DRE. Overall, patients with a positive DRE also had a higher rate of cancer detection than those with a negative DRE.

In the present patients only three of the 23 (13\%) who had a RP had pathologically insignificant disease. Using a slightly different definition of insignificant disease, Epstein et al. [18] found that 16\% of 157 men who had a RP after conventional biopsy for clinical stage T1c prostate cancer had pathologically insignificant disease. They defined pathological insignificance as a Gleason score of $<7$, tumour volume $<0.2 \mathrm{~mL}$ and tumour confined to the prostate. In their study, the number of biopsy cores was not standardized, but had a mean and median of five cores per patient. Given that more cores were obtained in the present study (12), more insignificant disease would be expected to be detected, yet we detected a smaller percentage of insignificant disease. This might reflect that the conventional technique over-samples small foci of cancer, i.e. that the same region 
was sampled repeatedly, while the template technique ensures that each region is sampled only once. An alternative reason for the difference might simply be that the present study only examined 23 patients who had RP and a larger study would show no difference from previous work.

We assessed the ability of template-guided biopsy to predict the RP Gleason score, and found that it led to accurate grading in most patients (52\%), over-grading in two (9\%), under-grading in eight (35\%), and in one patient with Gleason 6 disease there was insufficient malignant tissue obtained on biopsy to assign a Gleason score. Mian et al. [19] studied 205 men who had had a traditional extended biopsy before RP, and found a concordance rate of 68\%, undergrading by biopsy in 17\% and over-grading in $15 \%$. Again perhaps, the concordance rate in the present study might be lower because there were too few patients. However, a recent ex vivo comparison of the TargetScan device and conventional biopsy showed that the template-guided biopsy more accurately predicted RP Gleason score than traditional TRUS biopsy [3].

This device has various potential advantages over traditional TRUS-guided biopsy. As the probe remains fixed during the procedure, it minimizes image distortion due to probe movement against the prostate. Its capacity to map the prostate and to record the exact location of each core has several important potential advantages. For patients undergoing second biopsies, the pattern can be altered to examine under-sampled regions and suspicious locations can be precisely rebiopsied. Last, the instrument can be used for planned template biopsy, targeted biopsy and saturation biopsy.

While the TargetScan system has shown several improvements over traditional TRUSguided biopsy, there are several advances that could further improve its utility for prostate cancer diagnosis in the future. The current biopsy protocol arrays 12 cores geometrically throughout the prostate, without consideration for the non-random distribution of cancer throughout the prostate, or the need for more cores in larger prostates [20]. Alternative algorithms that adjust for the non-random distribution of tumour within the prostate, and alter the number of cores based on gland volume, could improve the diagnostic ability.
There are several limitations to the present study. It was a retrospective chart review and there is no direct comparison between this device and traditional TRUS-guided biopsy. We attempted to minimize the bias of a retrospective study by collecting objective data except for the DRE findings, which were primarily collected from a document completed by the treating physician at the time of biopsy. Our definition of tumour volume was a percentage and not an absolute number. This is again due to the retrospective nature of the analysis. Prostate tumour volumes at our institutions are reported in this manner. However, a 20\% tumour volume for a $20 \mathrm{~g}$ prostate would correspond to $4 \mathrm{~g}$ of cancer, which exceeds a volume of $0.5 \mathrm{~mL}$. We therefore think that the 20\% reference point is a conservative estimate of low tumour volume.

In conclusion, traditional TRUS-guided prostate biopsy technique imprecisely locates the biopsy sites and as a result might not accurately describe the size, location and grade of the tumour, all of which are important in determining the optimum treatment for patients diagnosed with prostate cancer. Transrectal template biopsy offers several advantages over the traditional biopsy, including the ability to uniformly distribute biopsies throughout the prostate and record the location from which biopsy cores were taken, allowing re-biopsy of specific locations of interest in the future. We have shown that the precision of a templateguided approach potentially leads to higher cancer detection rates, without increasing the rate of detection of pathologically insignificant disease.

\section{CONFLICT OF INTEREST}

Gerald L. Andriole is a Stock Holder for Mentioned Product. John T. Wei, Adam S. Kibel, Thomas J. Polascik and Samir Taneja are Study Investigators Funded by Sponsor.

\section{REFERENCES}

1 Jemal A, Siegel R, Ward E et al. Cancer statistics, 2007. CA Cancer J Clin 2007; 57 : 43-66

2 Tarone RE, Chu KC, Brawley OW. Implications of stage-specific survival rates in assessing recent declines in prostate cancer mortality rates. Epidemiology 2000; 11: 167-70
3 Andriole GL, Bullock TL, Belani JS et al. Is there a better way to biopsy the prostate? Prospects for a novel transrectal systematic biopsy approach. Urology 2007; 70: 22-6

4 Loch T. Urologic imaging for localized prostate cancer in 2007. World J Urol 2007; 25: 121-9

5 Freedland SJ, Aronson WJ, Csathy GS et al. Comparison of percentage of total prostate needle biopsy tissue with cancer to percentage of cores with cancer for predicting PSA recurrence after radical prostatectomy: results from the SEARCH database. Urology 2003; 61: 742-7

6 Presti JC Jr. Prostate biopsy: how many cores are enough? Urol Oncol 2003; 21 : 135-40

7 Presti JC Jr, Chang JJ, Bhargava V, Shinohara K. The optimal systematic prostate biopsy scheme should include 8 rather than 6 biopsies: results of a prospective clinical trial. J Uro/2000; 163 : 163-6

8 Presti JC Jr, O'Dowd GJ, Miller MC, Mattu R, Veltri RW. Extended peripheral zone biopsy schemes increase cancer detection rates and minimize variance in prostate specific antigen and age related cancer rates: results of a community multi-practice study. J Urol 2003; 169: 125-9

9 Raaijmakers R, Kirkels WJ, Roobol MJ, Wildhagen MF, Schroder FH. Complication rates and risk factors of 5802 transrectal ultrasound-guided sextant biopsies of the prostate within a population-based screening program. Urology 2002; 60: 826-30

10 Siddiqui EJ, Ali S, Koneru S. The rectal administration of lignocaine gel and periprostatic lignocaine infiltration during transrectal ultrasound-guided prostate biopsy provides effective analgesia. Ann $R$ Coll Surg Eng/ 2006; 88: 218-21

11 de Jesus CM, Correa LA, Padovani CR. Complications and risk factors in transrectal ultrasound-guided prostate biopsies. Sao Paulo Med J 2006; 124 : 198-202

12 Etzioni R, Penson DF, Legler JM et al. Overdiagnosis due to prostate-specific antigen screening: lessons from U.S. prostate cancer incidence trends. J Natl Cancer Inst 2002; 94: 981-90

13 Kozlowski P, Chang SD, Jones EC, Berean KW, Chen H, Goldenberg SL. Combined diffusion-weighted and dynamic contrast-enhanced MRI for 
prostate cancer diagnosis - correlation with biopsy and histopathology. J Magn Reson Imaging 2006; 24: 108-13

14 Kumar V, Jagannathan NR, Kumar R et al. Transrectal ultrasound-guided biopsy of prostate voxels identified as suspicious of malignancy on threedimensional (1)H MR spectroscopic imaging in patients with abnormal digital rectal examination or raised prostate specific antigen level of $4-10 \mathrm{ng} / \mathrm{mL}$. $\mathrm{N}$ MR Biomed 2007; 20: 11-20

15 Gore JL, Shariat SF, Miles BJ et al. Optimal combinations of systematic sextant and laterally directed biopsies for the detection of prostate cancer. J Urol 2001; 165: 1554-9
16 Guichard G, Larre S, Gallina A et al. Extended 21-sample needle biopsy protocol for diagnosis of prostate cancer in 1000 consecutive patients. Eur Urol 2007; 52: 430-5

17 Naughton CK, Miller DC, Mager DE, Ornstein DK, Catalona WJ. A prospective randomized trial comparing 6 versus 12 prostate biopsy cores: impact on cancer detection. J Urol 2000; 164: 38892

18 Epstein Jl, Walsh PC, Carmichael M, Brendler CB. Pathologic and clinical findings to predict tumor extent of nonpalpable (stage T1c) prostate cancer. JAMA 1994; 271: 368-74

19 Mian BM, Lehr DJ, Moore CK et al. Role of prostate biopsy schemes in accurate prediction of Gleason scores. Urology 2006; 67: 379-83

20 Remzi M, Fong YK, Dobrovits $M$ et al. The Vienna Nomogram. Validation of a novel biopsy strategy defining the optimal number of cores based on patient age and total prostate volume. J Urol 2005; 174 : 1256-60

Correspondence: Adam S. Kibel, Washington University School of Medicine, 4960 Children's Place, Box 8242, St Louis, M0 63105, USA.

e-mail:kibela@wustl.edu

Abbreviation: $\mathrm{RP}$, radical prostatectomy. 